\title{
Non-Cascaded Short-Term Pumped-Storage Hydro- Thermal Scheduling Using Accelerated Particle Swarm Optimization
}

\author{
Muhammad Salman Fakhar \\ Syed Abdul Rehman Kashif \\ Muhammad Asghar Saqib \\ Farhan Mehmood \\ Department of Electrical Engineering. \\ University of Engineering and Technology \\ Lahore, Pakistan. \\ salmanfakhar@uet.edu.pk
}

\begin{abstract}
This paper presents the implementation of a variant of the famous particle swarm optimization, known as Accelerated Particle Swarm Optimization (APSO), on a non-cascaded or a two-unit hydro-thermal system with consideration of hydal pumping in light loading intervals of hydro-thermal scheduling period. APSO is an easy to program and easy to implement variant of Particle Swarm Optimization (PSO) that has the ability to converge to a good approximate to global optimum within a few iterations. A standard pumped-storage hydrothermal scheduling problem, discussed in existing literature, is considered for the implementation of APSO. A comparison of this implementation is also given with the previously existing implementations of other algorithms.
\end{abstract}

Index Terms-Accelerated PSO, Hydro-thermal scheduling, Pumped-storage

List of symbols and abbreviations

\begin{tabular}{|l|l|}
\hline APSO & Accelerated Particle Swarm Optimization \\
\hline$f$ & Cost function \\
\hline$n_{j}$ & $\begin{array}{l}\text { Number of hours in } j^{\text {th }} \text { interval } \\
\text { interval }\end{array}$ \\
\hline$F_{j}$ & Water discharge rate at $j^{\text {th }}$ interval \\
\hline$D_{j}$ & $\begin{array}{l}\text { Total discharge rate at the end of all } \\
\text { scheduling intervals }\end{array}$ \\
\hline$D_{t o t}$ & Volume of water in reservoir at $j^{\text {th }}$ interval \\
\hline$V_{j}$ & Thermal power at $j^{\text {th }}$ interval \\
\hline$P_{s, j}$ & Hydro power at $j^{\text {th }}$ interval \\
\hline$P_{h, j}$ & Water Inflow in reservoir at $j^{\text {th }}$ interval \\
\hline$R_{j}$ & \\
\hline
\end{tabular}

\author{
Hafiz Zaheer Hussain \\ Department of Electrical Engineering. \\ NESPAK \\ Lahore, Pakistan.
}

\begin{tabular}{|l|l|}
\hline$S_{j}$ & Water spillage from reservoir at jth interval \\
\hline$\vartheta_{i}^{j}$ & Velocity of particle $i$ at iteration $j$ \\
\hline$P_{i}^{j}$ & Local best particle $i$ at iteration $j$ \\
\hline$P_{g}^{j}$ & Global best particle at iteration $j$ \\
\hline$X_{i}^{j}$ & Position of particle $\mathrm{i}$ at iteration $j$ \\
\hline
\end{tabular}

\section{INTRODUCTION}

Hydro-thermal scheduling is a nonlinear economic dispatch problem usually multi-modal in nature i.e. having multiple numbers of optima. References [1], [2] presents different heuristic and non-heuristic techniques that have already been implemented on hydro-thermal scheduling problem without considering pumping of water back to the reservoir. References [3], [4], specifically discuss various variants of particle swarm optimization (PSO) implemented on nonpumped hydro-thermal scheduling problem. According to the reference [3] on the PSO variants, for implementations on hydro-thermal scheduling, the superiority of PSO variants for such economic dispatch problems is well justified. However, for the non-cascaded and pumped hydro-thermal scheduling problems, the PSO implementations have not been reported yet. References [4] and [5] give the implementations of gradient search method and evolutionary programming on a pumped-storage hydro-thermal scheduling problem.

This paper gives the implementation of a fast-converging, easy to program and easy to implement variant of PSO known as accelerated PSO (APSO), as given in [6] and [7], on the problem mentioned in [4] and [5]. References [7]-[12] also presented the brilliant performance of accelerated PSO algorithm on various kinds of non-linear optimization problems in the domain of electrical engineering.

\section{Pumped Storage Hydro-Thermal Scheduling}

According to [4], [5], non-cascaded short-term pumpedstorage hydro-thermal scheduling is a type of short-term scheduling problem in which pumping of water is done back to the reservoir in the time intervals in which the load demand 
is quite low as compared to the other intervals of the scheduling; the intervals are known as the off-peak loading intervals. In these intervals, the pumping of water is made back to the reservoir while in the peak loading hours the generation of power is made by thermal and hydel plants collectively in an optimum way. So, the selected problem can be considered as an optimization problem having two sub problems, i.e. pumping of water in the low load hours and economic dispatch of the hydel and thermal plants optimally in the peak loading hours when generation is made from both types of synchronized plants. Mathematically, this problem can be described as in [4] and [5].

$\min (f)=\sum_{j=1}^{n} n_{j} F_{j}$

where $n_{j}$ is the number of hours in interval " $j$ " of the total scheduling period of one day to one week, $F_{j}$ is the cost of running the thermal power plant and " $f$ " is the total cost of all the intervals.

The discharge rate constraints, volume of water constraints, hydel power and thermal power constraints are given in equations (2) to (4) respectively.

$$
\begin{aligned}
& \sum_{j}^{N} n_{j} D_{j}=D_{\text {tot }} \\
& V_{\text {min }}<V_{j}<V_{\text {max }}
\end{aligned}
$$

$$
\left\{\begin{array}{lc}
D_{\min }<D_{j}<D_{\max } & \text { (water discharge limits) } \\
P_{s, \text { min }}<P_{s, j}<P_{S, \max } & \text { (Thermal power limits) } \\
P_{h, \text { min }}<P_{h, j}<P_{h, \max } & \text { (hydal power limits) }
\end{array}\right.
$$

The volume of water in the reservoir at any interval " $j$ " can be calculated using the continuity equation (5), as mentioned in reference $[5,6]$.

$V_{j}=V_{j-1}+n_{j}\left(R_{j}-D_{j}-S_{j}\right)$

It must be kept in mind that the pumped-storage problem consists of two sub problems as mentioned earlier. The difference comes into play in "(5)", while the value of $D_{j}$ can be positive or negative depending on whether the interval is pumping or non-pumping. $D_{j}$ will be positive for the nonpumping interval while its magnitude will be negative for the pumping interval.

\section{PROBLEM STATEMENT}

A non-cascaded pumped-storage hydro plant is to be operated in synchronism with a steam power plant to meet the load demands as given in Table 1. The generation and pumping characteristics of the selected pumped storage hydro plant are defined by the following equations:

\section{Generation characteristics:}

$$
Q\left(\mathrm{P}_{H}\right)=2 \mathrm{P}_{H} \text { acre }-f t / h \text { for } 0 \leq \mathrm{P}_{H} \leq+300 \mathrm{MW}
$$

\section{Pumping characteristics:}

$Q\left(\mathrm{P}_{P}\right)=-600$ acre $-f t / h$ with $\mathrm{P}_{P}=-300 \mathrm{MW}$

The steam power plant has the following cost characteristics: $F\left(\mathrm{P}_{S}\right)=3877.5+3.9795 \mathrm{P}_{S}+0.00204 P_{S}^{2} \$ / \mathrm{h}$ for $200 M W \leq P_{S} \leq 2500 M W(8)$

The load characteristics are given in Table 1 and this standard problem is taken from [4] and [5].

\section{OptimizATION Methodology: ACCELERATED PSO}

The canonical particle swarm optimization is a heuristic optimization algorithm in which the solutions of the objective function are randomly started and named as "particles". The particles are then updated to better values iteratively by using the PSO velocity and position updating "(9)".

TABLE 1: LOAD CHARACTERISTICS. EACH PERIOD IS OF 4 HOURS LENGTH.

\begin{tabular}{|c|c|c|c|c|c|c|}
\hline Period & 1 & 2 & 3 & 4 & 5 & 6 \\
\hline $\begin{array}{c}\text { Load } \\
(\mathrm{MW})\end{array}$ & 1600 & 1800 & 1600 & 500 & 500 & 500 \\
\hline
\end{tabular}

$\left\{\begin{array}{l}\vartheta_{i}^{j+1}=R\left(\vartheta_{i}^{j}+\operatorname{Rand}\left(0, \frac{\phi}{2}\right) \cdot\left(P_{i}^{j}-X_{i}^{j}\right)+\operatorname{Rand}\left(0, \frac{\phi}{2}\right) \cdot\left(P_{g}^{j}-X_{i}^{j}\right)\right) \\ X_{i}^{j+1}=X_{i}^{j}+\vartheta_{i}^{j+1}\end{array}\right.$

It can be seen in "(9)" that the updating of particle $X_{i}{ }^{j}$ depends upon the update of velocity first which has dependence upon current global best term $P_{g}^{j}$ and current local best term $P_{i}^{j}$ at iteration $j$. According to reference [7], the current local best term is used to enhance the diversity in quality solution, which is not oppressively desired if the optimization problem is not highly non-linear and multimodal. Moreover, this velocity update equation adds to the programming complexity and the emulators take more time for the bigger problems to emulate. This velocity update step can be omitted from the canonical PSO equation and a single step equation can be utilized for particles update to increase the convergence rate. The resultant equation is referred as single equation form of accelerated PSO as given in [7]. It can be observed, in the following equation, that the dependency on current local best is no more included to solve simple nonlinear equations.

$$
\left\{X_{i}^{j+1}=(1-\beta) X_{i}^{j}+(\beta)\left(\mathrm{P}_{g}^{j}\right)+(\alpha)(\varepsilon-0.5)\right.
$$

where $\beta$ is $0.5, \alpha=0.2$, and $\varepsilon$ is a random variable which can have any value from 0 to 1 as given in [7].

\section{A. Steps to Implement APSO on the Problem of Interest}

To implement APSO on "(6)" to "(8)", following will be the steps: 
1. Initialize the volume vectors (particles) within the given constraints, randomly.

2. Calculate the discharge rate, hydel power and thermal power using the values in step 1 .

3. Check the constraints. If the constraints are not met, restart from step 1. If the constraints are met, proceed to step 4.

4. Find the cost using thermal power values found in step 2.

5. Find the minimum cost value and the corresponding volume vector. That will be the global best vector.

6. Update all the particles using APSO updating "(10)".

7. Iterate from steps 2 to 6 till the stopping criterion is reached.

8. Print the results

\section{RESULTS AND DISCUSSION}

The accelerated PSO is implemented on the test problem of non-cascaded pumped-storage hydro-thermal scheduling, as given in "(6)" to "(8)", and already discussed in the [5] and [6]. The convergence characteristics and results of final iteration of the implementation are presented in Figure 1 and Table 2 respectively.

The final optimized results are the same as that presented by [4] using gradient search algorithm. Reference [5] has shown better results while implementing Evolutionary programming algorithm. However, in [5] the constraints of discharge rate and hydropower are violated. The discharge rate must not exceed 800 acre-ft/hour as given in the problem, and the hydel power is limited to the maximum of $300 \mathrm{MW}$. The final results cannot be better than as presented by reference [4], to keep all the constraints.

The improvement observed with accelerated PSO is that the convergence rate is very high as compared to other implementations of non-cascaded hydro-thermal scheduling problems. The algorithm is fast in implementation and is easy to program as well, as compared to the other variants of PSO and other heuristic optimization algorithms. To emphasis on the fast convergence rate and the ability to approaching towards global optimum solution, of accelerated PSO implementation on this problem, a statistical analysis is also done and is presented in the form of bar charts in Figure 2.

Figures 2 and 3 present that the algorithm helps in obtaining a good approximate of the global optimum solution for most of the trials for 100 iterations and 200 iterations respectively. Tables 3 and 4 give the maximum, average and minimum values of final optimum values achieved in 50 trials of implementation. It can be observed in Figures 2 and 3 that the accelerated PSO mostly reaches the global optimum in the 50 trials experiment. This shows its ability to converge to the good approximations of the global optima. Figures 4 and 5 present the statistical analysis of the exact number of iterations in which the algorithm reaches the global optimum for every trial using 100 particles and 200 particles in a swarm respectively. It can be observed from Figures 4 and 5 that accelerated PSO gives high acceleration to reach towards the global optimum: mostly in less than 10 iterations, the optimum is achieved. This shows the superiority of accelerated PSO over other optimization techniques implemented on pumped storage or simple hydro-thermal scheduling problems as per the rate of convergence is concerned. Tables 5 and 6 give the statistical results of the number of iterations in which the global optimum is achieved by APSO algorithm in 50 trials when swarms of 100 and 200 particles are taken respectively. This depicts that the APSO algorithm has very fast convergence rate and on average it takes around 8 iterations to reach the global optimum solution in this pumped-storage hydro-thermal scheduling problem.

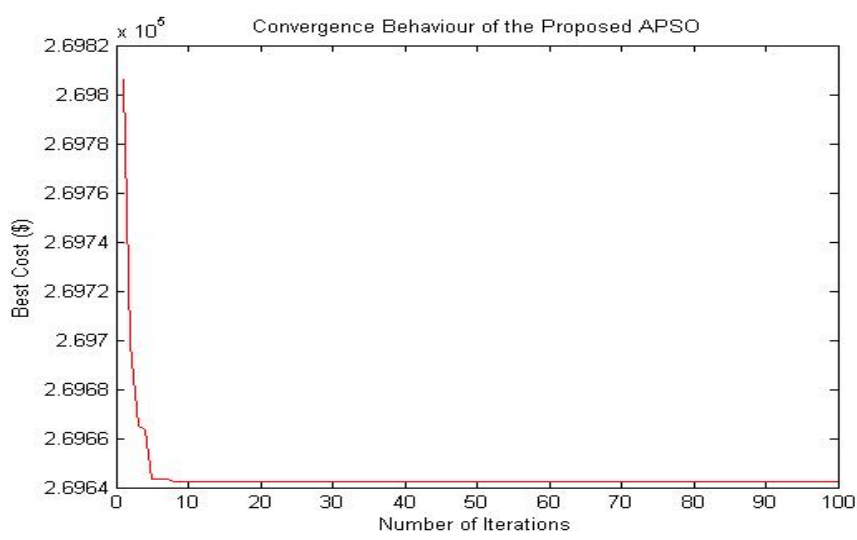

Figure1: Convergence characteristics of the proposed APSO implementation on pumped storage hydrothermal scheduling problem using swarm size of either 100 or 200 particles.

TABLE 2: BEST RESULT OF FINAL ITERATION OF APSO IMPLEMENTATION ON PUMPED STORAGE HYDRO-THERMAL SCHEDULING PROBLEM USING EITHER 100 or 200 PARTICLES' SWARM

\begin{tabular}{|c|c|c|c|c|c|c|}
\hline $\begin{array}{l}\text { Inter- } \\
\text { vals }\end{array}$ & $\begin{array}{l}\text { Demand } \\
\text { (MW) }\end{array}$ & $\begin{array}{c}\text { Ps } \\
\text { (MW) }\end{array}$ & $\begin{array}{c}\mathrm{Ph} \\
(\mathrm{MW})\end{array}$ & $\begin{array}{c}\mathrm{Q} \\
(\mathrm{ac}- \\
\mathrm{ft} / \mathrm{hr}) \\
\end{array}$ & $\mathrm{V}(\mathrm{ac}-\mathrm{ft})$ & Cost (\$) \\
\hline 1 & 1600 & 1449.99 & 150 & 500 & 5999.95 & \multirow{6}{*}{269642.4} \\
\hline 2 & 1800 & 1500 & 300 & 800 & 2799.819 & \\
\hline 3 & 1600 & 1450 & 159.97 & 500 & 800 & \\
\hline 4 & 500 & 800 & -300 & -600 & 3200 & \\
\hline 5 & 500 & 800 & -300 & -600 & 5600 & \\
\hline 6 & 500 & 800 & -300 & -600 & 8000 & \\
\hline
\end{tabular}

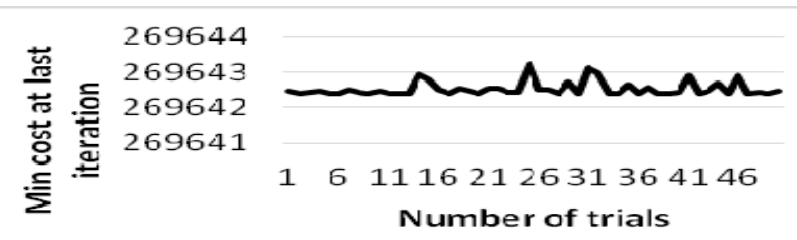

Figure 2: Minimum costs obtained in the final iteration for 50 iterations taking a swarm of 100 particles.

TABLE 3: STATISTICAL RESULTS OF GLOBAL OPTIMUM VALUE FOR THE 50 TRIALS FOR SWARM OF 100 PARTICLES.

\begin{tabular}{|c|c|c|}
\hline Max. cost (\$) & Average cost (\$) & Min. cost (\$) \\
\hline 269643.2336 & 269642.5318 & 269642.4001 \\
\hline
\end{tabular}


In comparison, reference [6] gives this implementation with evolutionary programming algorithm. It has achieved the convergence in more than 150 iterations. So, as presented in this paper, APSO has achieved very fast convergence rate as compared to the previous implementations of the different algorithms on the same problem.

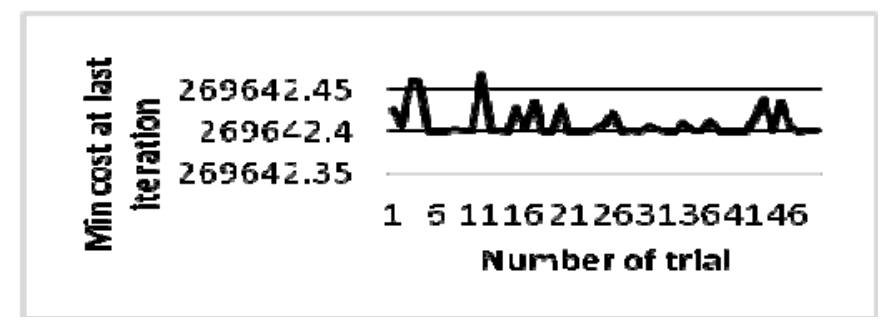

Figure 3: Minimum costs obtained in the final iteration for 50 iterations taking a swarm of 200 particles.

TABLE 4: STATISTICAL RESULTS OF GLOBAL OPTIMUM VALUE FOR THE 50 TRIALS FOR SWARM OF 200 PARTICLES.

\begin{tabular}{|c|c|c|}
\hline Max. cost & Average cost & Min. cost \\
\hline 269642.467 & 269642.41 & 269642.4 \\
\hline
\end{tabular}

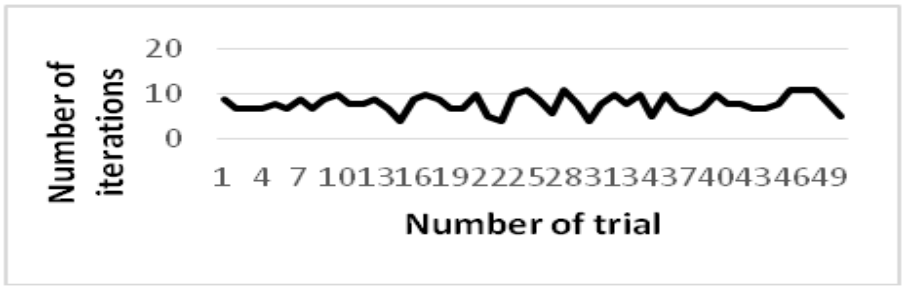

Figure 4: Numbers of iterations in which minimum cost is obtained in 50 trials considering swarm of 100 particles.

TABLE 5: STATISTICAL RESULTS OF NUMBER OF ITERATIONS TO REACH GLOBAL OPTIMUM FOR 50 TRIALS CONSIDERING SWARM OF 100 PARTICLES.

\begin{tabular}{|c|c|c|}
\hline $\begin{array}{c}\text { Max. } \\
\text { iteration }\end{array}$ & $\begin{array}{c}\text { Average } \\
\text { iteration }\end{array}$ & $\begin{array}{c}\text { Min. } \\
\text { iteration }\end{array}$ \\
\hline 11 & 8.2 & 4 \\
\hline
\end{tabular}

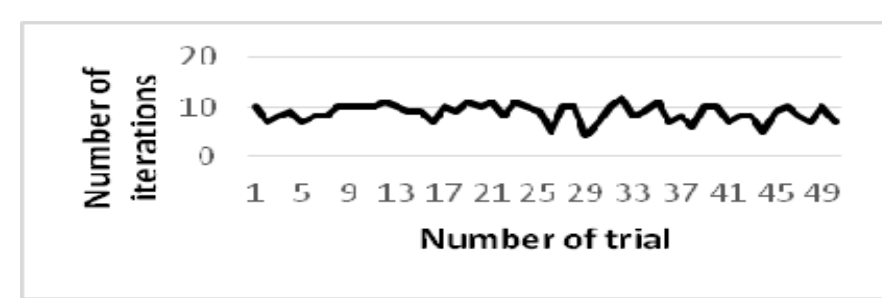

Figure 5: Numbers of iterations in which minimum cost is obtained in 50 trials considering swarm of 200 particles.
TABLE 6: STATISTICAL RESULTS OF NUMBER OF ITERATIONS TO REACH GLOBAL OPTIMUM FOR 50 TRIALS CONSIDERING SWARM OF 200 PARTICLES

\begin{tabular}{|c|c|c|}
\hline $\begin{array}{c}\text { Max. } \\
\text { iteration }\end{array}$ & $\begin{array}{c}\text { Average } \\
\text { iteration }\end{array}$ & $\begin{array}{c}\text { Min. } \\
\text { iteration }\end{array}$ \\
\hline 12 & 8.76 & 4 \\
\hline
\end{tabular}

\section{CONCLUSIONS}

A standard problem of non-cascaded pumped-storage shortterm hydro-thermal scheduling is solved using a fast converging and easy to program variant of PSO, known as accelerated PSO. It has been observed that the convergence rate is quite enhanced as compared to the previous implementations that are given in literature. Some errors were also observed in those implementations which remained unnoticed previously. Accelerated PSO should be a preferred variant of PSO for the implementation on multimodal problems like hydro-thermal scheduling where fast convergence and good robust approximations to global optimum solutions are desired.

\section{REFERENCES}

[1] S. Padmini and C. Rajan, "Improved PSO for short term hydrothermal scheduling”, Proceedings of International Conference on Sustainable Energy and Intelligent Systems, (SEISCON 2011), 2011, pp. 332-334.

[2] C. Samudi, G. Das, P. Ojha, T. Sreeni, and S. Cherian, "Hydro thermal scheduling using particle swarm optimization", Proceedings of Transmission and Distribution Conference and Exposition, 2008. T\&\# x00026; D. IEEE/PES, pp. 1-5.

[3] M.S. Fakhar et al. "Non cascaded short-term hydro-thermal scheduling using fully-informed particle swarm optimization", International Journal of Electrical Power \& Energy Systems 73 (2015), pp. 983-990.

[4] A.J. Wood and B. F. Wollenberg, Power generation, operation, and control: John Wiley \& Sons, 2012.

[5] S.K. Khandualo, A.K. Barisal and P.K. Hota, "Scheduling of pumped storage hydrothermal system with evolutionary programming", Journal of Clean Energy Technologies 1.4 (2013), pp. 308-312.

[6] X.S. Yang, Engineering optimization: an introduction with metaheuristic applications, John Wiley \& Sons, 2010, pp. 203219.

[7] X.S. Yang, S. Deb and S. Fong, "Accelerated particle swarm optimization and support vector machine for business optimization and applications", in: NDT2011, CCIS 136, Springer, 2011, pp. 53-66.

[8] A.H. Gandomi, G.J. Yun, X.S. Yang, and S. Talatahari, "Combination of chaos and accelerated particle swarm optimization", Communications in Nonlinear Science and Numerical Simulations, 18, 2013, pp. 327-340.

[9] A. El-Fergany, "Accelerated particle swarm optimization-based approach to the optimal design of substation grounding grid", Przegląd elektrotechniczny, ISSN 0033-2097, R. 89 NR 7/2013.

[10] S. Talatahari, E. Khalili and S.M. Alavizadeh, "Accelerated particle swarm for optimum design of frame structures", Hindawi Publishing Corporation Mathematical Problems in Engineering, vol. 2013, Article ID 649857.

[11] K. Prajna, G.S.B. Rao, K.V.V.S. Reddy and R.U. Maheswari, "A new dual channel speech enhancement approach based on accelerated particle swarm optimization (APSO)", I.J. Intelligent 
Systems and Applications, March 2014, vol. 04, pp. 1-10, DOI: 10.5815/ijisa. 2014.04.01.

[12] I. Rahman, P.M. Vasant, B. Singh, M. Singh and M.A. AlWadud, "On the performance of accelerated particle swarm optimization for charging plug-in hybrid electric vehicles", Elsevier Alexandria Engineering Journal, vol. 55, (2016), pp. 419-426. 